\title{
Correction to: Surface anchorage of superantigen SEA promotes induction of specific antitumor immune response by tumor-derived exosomes
}

\author{
Fangming Xiu ${ }^{1,2} \cdot$ Zhijian Cai $^{1} \cdot$ Yunshan Yang ${ }^{1} \cdot$ Xiaojian Wang ${ }^{1} \cdot$ Jianli Wang ${ }^{1} \cdot$ Xuetao Cao ${ }^{1,2}$
}

Published online: 15 January 2020

(C) Springer-Verlag GmbH Germany, part of Springer Nature 2020

Correction to: J Mol Med (2007) 85:511-521

https://doi.org/10.1007/s00109-006-0154-1

The corrected Figure 1 image and caption is presented in this paper.

The online version of the original article can be found at https://doi.org/ 10.1007/s00109-006-0154-1

$\triangle \quad$ Xuetao Cao

caoxt@immunol.org

Jianli Wang

jlwang@zju.edu.cn

1 Institute of Immunology, Zhejiang University, 353 Yan'an Road,

Hangzhou 310031, People's Republic of China

2 Institute of Immunology, Second Military Medical University, 800

Xiangying Road, Shanghai 200433, People's Republic of China 

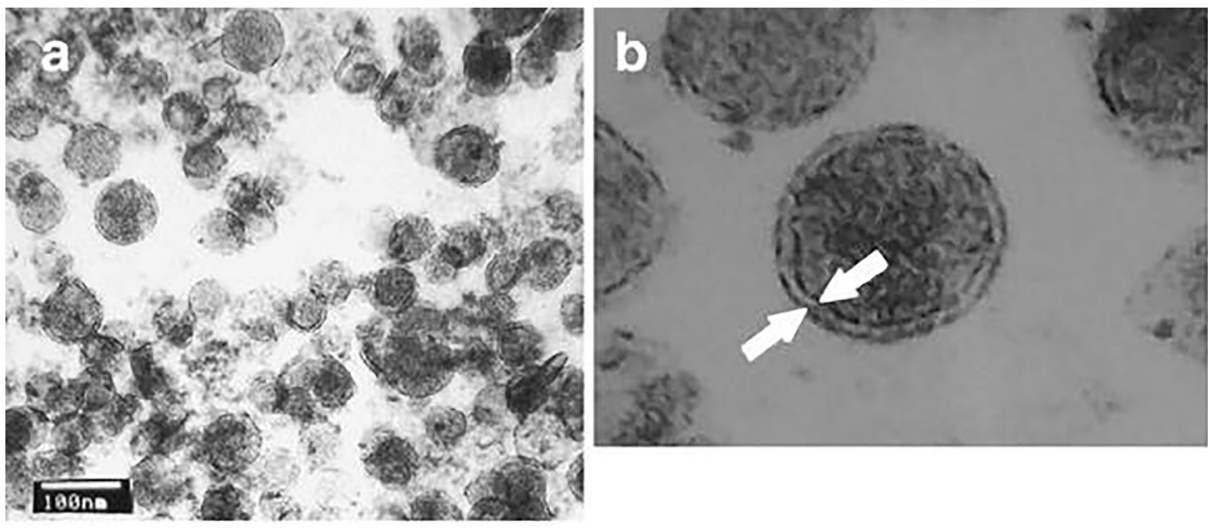

c

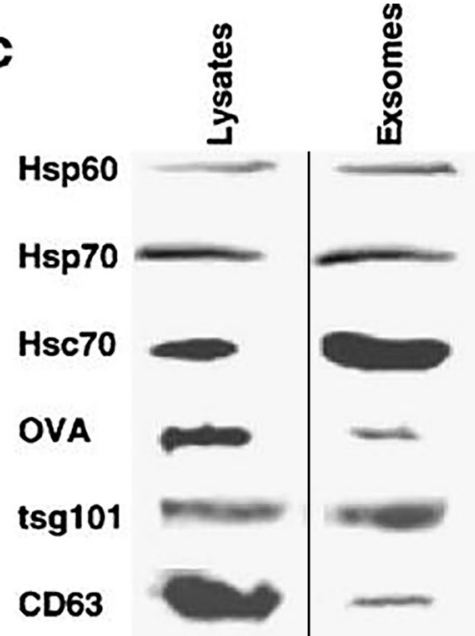

Fig. 1 Identification of E.G7-OVA-derived exosomes. Ultrathin sections were viewed under transmission electron microscope at $80 \mathrm{kv}$; the exosome sizes were from 40 to $100 \mathrm{~nm}$ and limited with a bilayer membrane. a Magnification: $\times 65,000$; b Magnification: $\times 100,000$, membrane structure (arrows) and the "honeycomb-like inner structure" are visible clearly. $\mathbf{c}$ Protein composition of exosomes derived from E.G7-
OVA cells by Western blot. Same amount of protein of E.G7-OVA cell lysates and exosomes were separated on 10\% SDS/PAGE, and the desired proteins were determined by their corresponding antibodies. Specific antibodies used here were antibodies for Hsp 60, Hsp70, Hsc70, OVA, tsg 101, and CD63. Line indicated the bands were not adjacent. The results are representative of three independent experiments 